\title{
Low Complexity Resource Allocation for Massive Carrier Aggregation
}

\author{
Stelios Stefanatos, Fotis Foukalas, Member, IEEE, and Theodoros A. Tsiftsis, Senior Member, IEEE
}

\begin{abstract}
Optimal resource allocation (RA) in massive carrier aggregation scenarios is a challenging combinatorial optimization problem whose dimension is proportional to the number of users, component carriers (CCs), and OFDMA resource blocks per CC. Towards scalable, near-optimal RA in massive CA settings, an iterative RA algorithm is proposed for joint assignment of CCs and OFDMA resource blocks to users. The algorithm is based on the principle of successive geometric programming approximations and has a complexity that scales only linearly with the problem dimension. Although its derivation is based on a relaxed formulation of the RA problem, the algorithm is shown to converge to integer-valued RA variables with probability 1 under mild assumptions on the distribution of user utilities. Simulations demonstrate improved performance of the proposed algorithm compared to commonly considered heuristic RA procedures of comparable complexity.
\end{abstract}

Index Terms-massive carrier aggregation, resource allocation, geometric programming, iterative algorithm, convergence.

\section{INTRODUCTION}

$\mathbf{C}$ ARRIER aggregation (CA) is considered as one of the key features of future cellular networks for effectively increasing the system bandwidth by simultaneous utilization of multiple component carriers (CCs) [1]. Although up to 5 CCs were initially considered for CA in the Long-Term-EvolutionAdvanced (LTE-A) standard, the increasing system demands strongly suggest that future applications of CA will utilize more CCs, towards realizing the concept of massive CA [2]. Proposals for CA of 32 CCs in LTE have appeared [3].

One of the major challenges of $\mathrm{CA}$ is the resource allocation (RA). With each CC typically consisting of multiple OFDMA resource blocks (RBs) and with limitations on the maximum number of CCs that UEs can utilize for communication, optimal RA becomes a constrained, integer optimization problem that is difficult to solve, even for a small number of available CCs [4], [5]. This has led to most RA proposals performing $\mathrm{CC}$ and $\mathrm{RB}$ allocation separately [4], [6], with $\mathrm{CC}$ allocation performed first by some heuristic method, followed by RB allocation per CC. Towards improving system performance, the problem of joint $\mathrm{CC}$ and $\mathrm{RB}$ allocation was considered in [5], [7], where iterative RA algorithms were proposed in an attempt to reduce the number of combinations required to be examined by a brute-force solution approach. However, the

S. Stefanatos is with the Industrial Systems Institute, Athena Research and Innovation Centre, Patras, Greece (e-mail: sstefanatos@isi.gr).

F. Foukalas is with the Industrial Systems Institute, Athena Research and Innovation Centre, Patras, Greece (e-mail: foukalas@isi.gr).

T. A. Tsiftsis is with the School of Engineering, Nazarbayev University, Astana 010000, Kazakhstan (e-mail: theodoros.tsiftsis@ @u.edu.kz).

This work has been supported by the "Spectrum Overlay through Aggregation of Heterogeneous Dispersed Bands" project, ICT-SOLDER, www.ictsolder.eu, FP7 grant agreement number 619687. complexity of these algorithms becomes impractical when a massive CA scenario is considered.

In this correspondence, the problem of joint $\mathrm{CC} / \mathrm{RB}$ allocation to UEs in a massive CA scenario (e.g., with 50 available CCs) is considered, with the goal of maximizing the weighted sum utility of the users. Towards obtaining an efficient, scalable RA algorithm, the method of successive geometric programming approximations $[\overline{8}]$ is applied to a relaxed formulation of the RA problem. The resulting iterative RA algorithm has a simple analytical representation (no general-purpose numerical optimization procedures are required) with its complexity scaling only linearly with the problem dimension when the maximum number of iterations is kept fixed. It is shown that, under mild assumptions on the distribution of utility functions, the algorithm converges with probability 1 to integer-valued RA variables, which, in certain special cases of the RA problem, are the optimal ones. Performance of the algorithm in a massive CA setting is investigated numerically, where it is shown to outperform heuristic RA approaches of comparable complexity.

\section{System Model and Problem Formulation}

The downlink or uplink of a single cell is considered that serves $K \geq 2$ user equipments (UEs), indexed by $k \in \mathcal{K} \triangleq$ $\{1,2, \ldots, K\}$. The system has $M \geq 2$ available CCs with transmissions over the $m$-th $\mathrm{CC}, m \in \mathcal{M} \triangleq\{1,2, \ldots, M\}$, performed via OFDMA. Without loss of generality, it will be assumed that all CCs have the same bandwidth that is partitioned into $N \geq 2 \mathrm{RBs}$ of equal size, indexed by $n \in \mathcal{N} \triangleq\{1,2, \ldots, N\}$.

Let $\phi_{k, m, n}>0$ denote the utility that the $k$-th UE achieves when utilizing the $n$-th RB of the $m$-th CC. A commonly used metric for resource allocation (RA) purposes is the weighted sum utility (WSU) of UEs [5], [7], [9], defined as

$$
\mathrm{WSU} \triangleq \sum_{k \in \mathcal{K}} w_{k} \sum_{m \in \mathcal{M}} \sum_{n \in \mathcal{N}} \alpha_{k, m, n} \phi_{k, m, n}
$$

where $w_{k}>0$ is the weight of $\mathrm{UE} k$, and $\left\{\alpha_{k, m, n}\right\}$ is a set of $K M N$ binary-valued $(0-1) \mathrm{RA}$ variables reflecting whether UE $k$ is allocated to RB $n$ of CC $m$ or not $\left(\alpha_{k, m, n}=1,0\right.$, respectively). With the goal of maximizing the WSU (equivalently, minimizing $1 / \mathrm{WSU}$ ), the RA variables are obtained as the solution of the constrained integer (binary) optimization problem described in 2), where $\mathcal{M}_{k} \triangleq\left\{m \in \mathcal{M}: \alpha_{k, m, n}=\right.$ 1 for some $n \in \mathcal{N}\}$ is the set of CCs where UE $k$ is allocated at least one RB and $|\mathcal{S}|$ denotes the number of elements of the set $\mathcal{S}$. 


$$
\left\{\begin{aligned}
& \operatorname{minimize} 1 / \mathrm{WSU}, \\
& \text { subject to } \mathrm{C} 1: \sum_{k \in \mathcal{K}} \alpha_{k, m, n} \leq 1, \forall m, n, \\
& \mathrm{C} 2:\left|\mathcal{M}_{k}\right| \leq M_{k}, \forall k, \\
& \mathrm{C} 3:\left|\cup_{k \in \mathcal{K}} \mathcal{M}_{k}\right| \leq M_{0}, \\
& \mathrm{C} 4: \alpha_{k, m, n} \in\{0,1\}, \forall k, m, n,
\end{aligned}\right\}
$$

Constraint $\mathrm{C} 1$ corresponds to the common requirement that at most one UE is allocated to the $n$-th RB of the $m$-th CC. Constraint C2 restricts the number of CCs used by UE $k$ to a maximum value $M_{k} \leq M$, possibly different among UEs. Constraint C 3 guarantees that $M_{0} \leq M$ CCs in total will be utilized by the system for RA purposes. The last two constraints may be imposed in practice due to, e.g., compatibility with legacy devices that can communicate only via a single $\mathrm{CC}$, power consumption considerations when a UE operates on multiple CCs at the same time, and utilization of the remaining $M-M_{0} \mathrm{CCs}$ for other system applications of lower priority.

For the special case where $M_{k}=M_{0}=M$ for all $k \in \mathcal{K}$, constraints C2 and C3 become irrelevant and the RA problem effectively corresponds to a standard OFDMA resource allocation problem on a single CC with $M N$ RBs [9]. The optimal allocation in this case is a simple, "winnertakes-all” assignment per RB [9], namely,

$$
\alpha_{k^{*}, m, n}= \begin{cases}1, & k^{*}=\arg \max _{k \in \mathcal{K}} w_{k} \phi_{k, m, n}, \\ 0, & k \neq k^{*},\end{cases}
$$

for all $m, n$, with ties resolved arbitrarily. This algorithm has a complexity that scales only linearly with number of RA variables. However, for the general case where limitations are imposed on the maximum number of employed CCs, optimal RA requires solving a combinatorial problem whose complexity scales exponentially with the number of RA variables. For massive CA applications, optimal solution of the RA allocation problem becomes impractical, which motivates the search for alternative RA procedures.

\section{Low-Complexity Resource Allocation ALGORITHM}

Towards obtaining an efficient, low complexity RA algorithm for massive CA scenarios, the original RA problem can be reformulated by introducing two sets of auxiliary RA variables, $\left\{\beta_{k, m}\right\},\left\{\gamma_{m}\right\}$ of cardinality $K M$, and $M$, respectively. The RA variables $\left\{\beta_{k, m}\right\}$ indicate whether UE $k$ is allocated to CC $m$ or not ( $\beta_{k, m}=1,0$, respectively) and the RA variables $\left\{\gamma_{m}\right\}$ indicate whether CC $m$ is utilized for transmissions by the system or not $\left(\gamma_{m}=1,0\right.$, respectively). By expressing the WSU in the equivalent form

$$
\mathrm{WSU}=\sum_{k \in \mathcal{K}} w_{k} \sum_{m \in \mathcal{M}} \gamma_{m} \beta_{k, m} \sum_{n \in \mathcal{N}} \alpha_{k, m, n} \phi_{k, m, n},
$$

and treating $\left\{\alpha_{k, m, n}\right\},\left\{\beta_{k, m}\right\},\left\{\gamma_{m}\right\}$ as independent optimization variables that are continuous-valued in the interval
$(0,1]$, a relaxed version of the original RA problem can be formulated as

$$
\left\{\begin{array}{c}
\text { minimize } 1 / \text { WSU, } \\
\text { subject to } \mathrm{C} 1: \sum_{k \in \mathcal{K}} \alpha_{k, m, n} \leq 1, \forall m, n, \\
\mathrm{C} 2^{\prime}: \sum_{m \in \mathcal{M}} \beta_{k, m} \leq M_{k}, \forall k, \\
\mathrm{C} 3^{\prime}: \sum_{m} \gamma_{m} \leq M_{0} \\
\mathrm{C}^{\prime}: \alpha_{k, m, n}, \beta_{k, m}, \gamma_{m} \in(0,1], \forall k, m, n .
\end{array}\right\}
$$

Note that the introduction of the auxiliary variables, although increasing the dimension of the problem as a total of $K M N+K M+M$ variables have to be found. However, it allows for expressing the combinatorial constraints C2 and C3 of the original RA problem formulation in the much more convenient, linear formulation of $\mathrm{C}^{\prime}$ and $\mathrm{C} 3^{\prime}$, respectively. Consideration of strictly positive values for the RA variables (even though they can be equal to zero, in principle) is only a technical requirement for the following algorithm development and has no effect in practice, since a RA variable of value less than a sufficiently small positive threshold may be safely assumed as zero.

Consideration of the relaxed RA problem is motivated by noting that its formulation corresponds to that of a convex, geometric programming (GP) problem [10], with the exception that the objective function is not a posynomial with respect to (w.r.t.) the RA variables, but the inverse of a posynomial. This exact type of problem was considered in [8] where an iterative solution algorithm was proposed based on solving a sequence of successive GP approximations of the problem formulation. The algorithm is guaranteed to converge to a point satisfying the Karush-Kuhn-Tucker (KKT) conditions of the problem.

Applying the same procedure as in [8] for solving the relaxed RA problem results in obtaining the (positive-valued) estimates $\left\{\alpha_{k, m, n}^{(i)}\right\},\left\{\beta_{k, m}^{(i)}\right\},\left\{\gamma_{m}^{(i)}\right\}$ of the RA variables at iteration $i$, as the solution of a modified version of the relaxed RA problem where the objective function 1/WSU is approximated by the monomial (see [8] for details)

$$
\tilde{f}^{(i)}=\prod_{k, m, n}\left(\frac{w_{k} \phi_{k, m, n} \beta_{k, m} \gamma_{m} \alpha_{k, m, n}}{u_{k, m, n}^{(i)}}\right)^{-u_{k, m, n}^{(i)}},
$$

where

$$
u_{k, m, n}^{(i)} \triangleq \frac{w_{k} \phi_{k, m, n} \beta_{k, m}^{(i-1)} \gamma_{m}^{(i-1)} \alpha_{k, m, n}^{(i-1)}}{\sum_{k^{\prime}, m^{\prime}, n^{\prime}} w_{k^{\prime}} \phi_{k^{\prime}, m^{\prime}, n^{\prime}} \beta_{k^{\prime}, m^{\prime}}^{(i-1)} \gamma_{m^{\prime}}^{(i-1)} \alpha_{k^{\prime}, m^{\prime}, n^{\prime}}^{(i-1)}},
$$

for all $k, m, n$, where $\left\{\alpha_{k, m, n}^{(i-1)}\right\},\left\{\beta_{k, m}^{(i-1)}\right\},\left\{\gamma_{m}^{(i-1)}\right\}$ are the estimates of the RA variables obtained at iteration $i-1$. As the following result shows, the new RA variables estimates can be obtained via a simple (semi-) closed form formula, with no need to employ general-purpose numerical solvers.

Lemma 1. The optimal RA variables for the GP optimization 


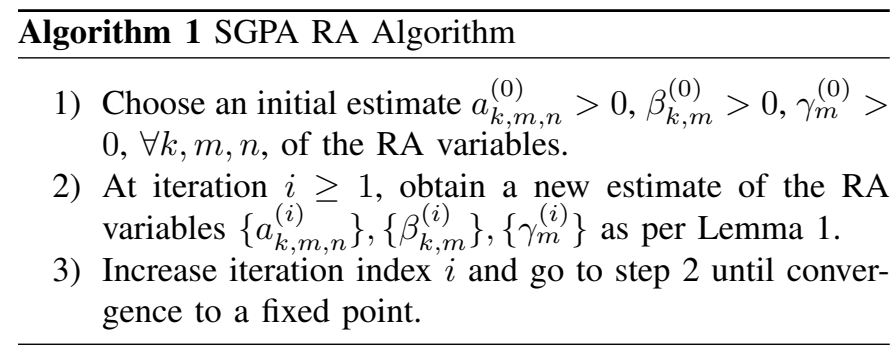

problem resulting by replacing the objective function 1/WSU in the relaxed $R A$ problem formulation with $\tilde{f}^{(i)}$ as defined in (6) equals

$$
\begin{aligned}
\alpha_{k, m, n}^{(i)} & =\alpha_{k, m, n}^{(i-1)} \frac{\beta_{k, m}^{(i-1)} w_{k} \phi_{k, m, n}}{\sum_{k^{\prime}} \alpha_{k^{\prime}, m, n}^{(i-1)} \beta_{k^{\prime}, m}^{(i-1)} w_{k^{\prime}} \phi_{k^{\prime}, m, n}} \\
\beta_{k, m}^{(i)} & =\min \left\{1, \beta_{k, m}^{(i-1)} \frac{\sum_{n} w_{k} \gamma_{m}^{(i-1)} a_{k, m, n}^{(i-1)} \phi_{k, m, n}}{\lambda_{k}^{(i)}}\right\}, \\
\gamma_{m}^{(i)} & =\min \left\{1, \gamma_{m}^{(i-1)} \frac{\sum_{k, n} w_{k} \beta_{k, m}^{(i-1)} a_{k, m, n}^{(i-1)} \phi_{k, m, n}}{\mu^{(i)}}\right\},
\end{aligned}
$$

for all $k \in \mathcal{K}, m \in \mathcal{M}, n \in \mathcal{N}$, where $\lambda_{k}^{(i)}>0, \mu^{(i)}>0$, are uniquely determined by the conditions $\sum_{m} \beta_{k, m}^{(i)}=M_{k}$, $\sum_{m} \gamma_{m}^{(i)}=M_{0}$, respectively.

Proof: Recalling that replacing the objective function of an optimization problem with its logarithm does not change the solution of the optimization variables [10], the objective $\tilde{f}^{(i)}$ can be replaced by $\log \left(\tilde{f}^{(i)}\right)$, which is equal to

$$
-\sum_{k, m, n} u_{k, m, n}^{(i)}\left(\log \left(a_{k, m, n}\right)+\log \left(\beta_{k, m}\right)+\log \left(\gamma_{m}\right)\right),
$$

after dropping additive constants that are independent of the RA variables and play no role in the solution. It can be verified that the resulting problem formulation is convex. Therefore, standard solution techniques using Lagrange multipliers can be employed [10] resulting in the optimal RA variables stated in the Lemma. Details are omitted.

The successive GP approximations (SGPA) RA algorithm is summarized at the top of the page. Its complexity scales only linearly with the number of RA variables, as long as the number of iterations required for convergence is independent of the dimension of the problem. Even though the latter condition is not the case, simulations show that limiting the number of iterations to a maximum number, irrespective of the dimension of the problem, yields good performance.

\section{Convergence Properties of the SGPA RA ALGORITHM}

Although the SGPA RA is guaranteed to converge to a KKT point of the relaxed RA problem formulation, it is of interest to determine further properties of the convergence in order to obtain insights on its operation and usefulness of provided solutions. The key to study the convergence is to view the parallel updates of the scalar RA variables performed by the algorithm as parallel updates of appropriate vector RA variables. In particular, consider (a) the RB allocation vector variables $\left\{\boldsymbol{\alpha}_{m, n}\right\}$, where $\boldsymbol{\alpha}_{m, n} \triangleq\left[\alpha_{1, m, n}, \alpha_{2, m, n}, \ldots, \alpha_{K, m, n}\right]$, (b) the UE-CC allocation vector variables $\left\{\boldsymbol{\beta}_{k}\right\}$, where $\boldsymbol{\beta}_{k} \triangleq$ $\left[\beta_{k, 1}, \beta_{k, 2}, \ldots, \beta_{k, M}\right]$, and (c) the CC activation vector variable $\gamma \triangleq\left[\gamma_{1}, \gamma_{2}, \ldots, \gamma_{M}\right]$. Using the compact notation $\mathbf{x}=$ $\left[x_{1}, x_{2}, \ldots, x_{P}\right]$ for representing any of the aforementioned vector variables, and according to Lemma 1 , the update rules of the SGPA RA algorithm are equivalent to updating the elements of $\mathbf{x}$ at iteration $i$ as

$$
x_{p}^{(i)}=\min \left\{1, x_{p}^{(i-1)} \frac{r_{p}^{(i)}}{\kappa^{(i)}}\right\}, p \in \mathcal{P} \triangleq\{1,2, \ldots, P\},
$$

where $r_{p}^{(i)}>0, p \in \mathcal{P}$, is independent of $\mathbf{x}^{(i)}$ and $\kappa^{(i)}>0$ is selected such that

$$
\sum_{p=1}^{P} x_{p}^{(i)}=L, \text { for all } i,
$$

where $L \in \mathcal{P}$. For example, with $\mathbf{x}$ corresponding to $\boldsymbol{\beta}_{k}$, for some $k \in \mathcal{K}$, index $p$ in $(8)$ represents the tuple $(k, m)$ with $m \in \mathcal{M}, \mathcal{P}=\mathcal{M}, P=M, \kappa^{(i)}=\lambda_{k}^{(i)}, L=M_{k}$, and $r_{p}^{(i)}=w_{k} \gamma_{m}^{(i-1)} \sum_{n} \alpha_{k, m, n}^{(i-1)} \phi_{k, m, n}$.

The following technical result is fundamental towards understanding the operation of the SGPA RA algorithm and its convergence properties.

Proposition 2. Let the iterations of (8) initialized by $\left\{x_{p}^{(0)}\right\}_{p \in \mathcal{P}}$, such that it holds $x_{p}^{(0)} \in(0,1]$, for all $p \in \mathcal{P}$, and assume that there exists a permutation $\pi$ of $\mathcal{P}$ and an integer $P_{0} \in \mathcal{P}$ such that $r_{\pi(1)}^{(i)}>r_{\pi(2)}^{(i)}>\cdots>r_{\pi\left(P_{0}\right)}^{(i)}>0$ and $r_{\pi\left(P_{0}+1\right)}^{(i)}=\cdots=r_{\pi(P)}^{(i)}=0$, for all $i \geq 0$. When the iterations are performed with finite (hardware) precision arithmetic, sequence $x_{p}^{(i)}, p \in \mathcal{P}$, converges to the limit

$$
\bar{x}_{p}=\left\{\begin{array}{ll}
1, & \text { if } r_{p}^{(0)}>0 \text { and } r_{p}^{(0)} \geq r_{\pi(L)}^{(0)}, \\
0, & \text { if } r_{p}^{(0)}=0 \text { or } r_{p}^{(0)}<r_{\pi(L)}^{(0)}
\end{array} .\right.
$$

Proof: See Appendix.

Remark: In case where $x_{p}^{(0)}=0$ for some $p \in \mathcal{P}$, it can be verified that $x_{p}^{(i)}=0$ for all $i \geq 0$, and the iterations of 8 are essentially performed for the sequence of indices in $\mathcal{P} \backslash p$ with Prop. 2 changed accordingly.

In order to obtain insights on the operation of the SGPA RA algorithm, consider the special case where $\left\{\alpha_{k, m, n}\right\}$ and $\left\{\gamma_{m}\right\}$ have been pre-selected (by external means) and the optimal $\left\{\beta_{k, m}\right\}$ are required. It is easy to see that in this case, the RA problem becomes a linear programming (LP) problem with a particularly simple solution [10]: Assign to UE $k \in \mathcal{K}$ the $M_{k}$ CCs corresponding to the largest effective CC utilities $\bar{\phi}_{k, m}, m \in \mathcal{M}$, where $\bar{\phi}_{k, m} \triangleq w_{k} \gamma_{m} \sum_{n} \alpha_{k, m, n} \phi_{k, m, n}$. Solving the same problem with the SGPA RA algorithm, results in the iterative estimates of $\left\{\beta_{k, m}\right\}$ for UE $k$ corresponding to the general update formula of 8 with $r_{k, m}^{(i)}=\bar{\phi}_{k, m}$ for all 


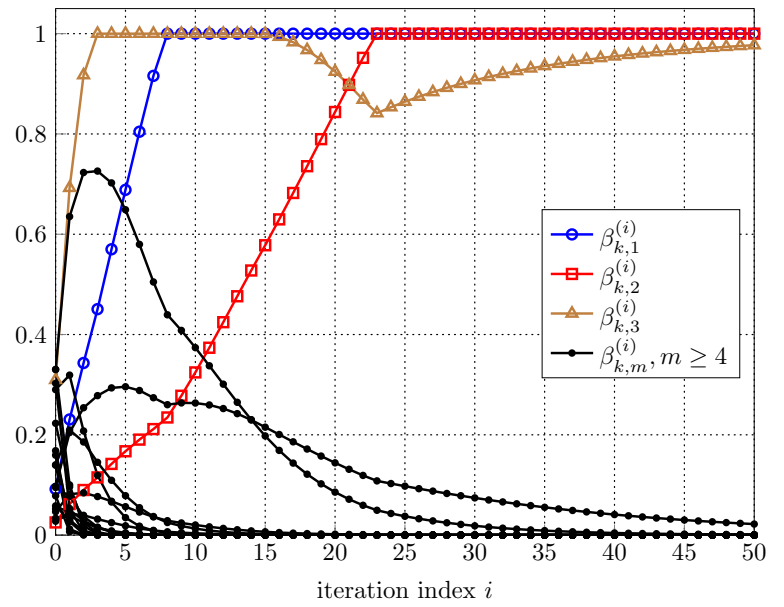

Fig. 1. Convergence example of the SGPA RA algorithm w.r.t. $\left\{\beta_{k, m}\right\}_{m \in \mathcal{M}}$ when $\left\{\alpha_{k, m, n}\right\}$ and $\left\{\gamma_{m}\right\}$ are given $\left(M=20, M_{k}=3\right)$.

$i$, which, by Prop. 2, converge to the optimal solution if the positive elements of $\left\{\bar{\phi}_{k, m}\right\}_{m \in \mathcal{M}}$ are not equal.

A typical example of the convergence of the $\left\{\beta_{k, m}\right\}_{m \in \mathcal{M}}$ estimates for a UE $k$ obtained by the SGPA RA algorithm in this special case is shown in Fig. 11, with $M=20$ and $M_{k}=3$. The effective weights $\left\{\bar{\phi}_{k, m}\right\}_{m \in \mathcal{M}}$ were randomly generated and ordered such that $\bar{\phi}_{k, 1}>\bar{\phi}_{k, 2}>\ldots>\bar{\phi}_{k, M}>0$, and a random initialization $\left\{\beta_{k, m}^{(0)}>0\right\}_{m \in \mathcal{M}}$ such that $\sum_{m \in \mathcal{M}} \beta_{k, m}=3$ was used. It can be seen that the RA variables converge to $\beta_{k, m}=1$ for $m=1,2,3$, as predicted by Prop. 2. Note that, even though at $i=50, \beta_{k, 3}$ has not achieved the value of 1 , stoping the iterations at $i=15$ and quantizing the RA variables to their nearest integer would still result in the optimal RA variables.

Although the above result demonstrates that the SGPA RA algorithm converges to the optimal RA variables under special cases, this is not the case in general, i.e., a suboptimal convergence point is expected. However, as the following result shows, the convergence point has desirable properties, namely, it is binary-valued irrespective of the initialization as long a mild condition on the utilities holds.

Proposition 3. Under that assumption that $\left\{\phi_{k, m, n}\right\}$ are distributed according to a joint continuous probability density function (p.d.f.) over $[0, \infty)^{K M N}$, and for any initialization of the $R A$ variables that is independent of $\left\{\phi_{k, m, n}\right\}$, the SGPA $R A$ algorithm converges with probability 1 to binary-valued $R A$ variables.

Proof: Since the SGP RA algorithm is guaranteed to converge to a KKT point of the relaxed RA problem, it follows that that the sequences $\left\{r_{p}^{(i)}\right\}_{p \in \mathcal{P}}$ of the general iteration formula of $(8)$ will converge to a limit $\left\{\bar{r}_{p}\right\}_{p \in \mathcal{P}}$. Assuming for the moment that no two positive elements of $\left\{\bar{r}_{p}\right\}_{p \in \mathcal{P}}$ are the same, it follows from fundamental properties of limits that for any sufficiently small $\epsilon>0$, there exists an iteration index $i_{\epsilon}$, a permutation $\pi$ of $\mathcal{P}$, and an integer $P_{0} \in \mathcal{P}$ such that it holds $\left|r_{p}^{(i)}-\bar{r}_{p}\right|<\epsilon$ for all $p \in \mathcal{P}$, with $r_{\pi(1)}^{(i)}>$ $r_{\pi(2)}^{(i)}>\cdots>r_{\pi\left(P_{0}\right)}^{(i)}>0, r_{\pi\left(P_{0}+1\right)}^{(i)}=\cdots=r_{\pi(P)}^{(i)}=0$, for all $i \geq i_{\epsilon}$. That is, as the algorithm approaches one of its fixed points, the elements of $\left\{r_{p}^{(i)}\right\}_{p \in \mathcal{P}}$, although varying in principle as the iterations progress, will achieve an ordering that holds for all $i \geq i_{\epsilon}$. Treating $\left\{x_{p}^{\left(i_{\epsilon}\right)}\right\}$ as an initialization point for the algorithm iterations, it follows from Prop. 2 that each RA variable will converge to either 0 or 1 .

Next, it will be shown that, with probability one, the limit $\left\{\bar{r}_{p}\right\}_{p \in \mathcal{P}}$ has no two positive elements that are equal. By examination of the equations corresponding to the KKT conditions of the relaxed RA problem, it can be verified that the condition $\bar{r}_{p}=\bar{r}_{q}$, for some $p \neq q$, does not hold identically (by default) at any KKT point. Noting that both the equations corresponding to the KKT conditions as well as the expressions for $r_{p}^{(i)}$ (and hence, $\bar{r}_{p}$ ) are posynomials w.r.t. the RA variables and $\left\{\phi_{k, m, n}\right\}$, it follows that the set $\mathcal{Z} \triangleq\left\{\left\{\phi_{k, m, n}\right\}: \bar{r}_{p}=\bar{r}_{q}>0\right.$, for some $\left.p \neq q\right\}$ is of (Lebesgue) measure zero [11, Corollary 10], which implies that the probability of the event $\left\{\phi_{k, m, n}\right\} \in \mathcal{Z}$ is zero.

Remark: The assumption of continuous-valued $\left\{\phi_{k, m, n}\right\}$ is critical for the operation and convergence of the proposed algorithm and applies to many possible utilities that can be considered for RA purposes including link capacity [5], [9]. The case of RA with discrete-valued utilities requires a different treatment.

\section{NUMERICAL EXAMPLES}

In order to demonstrate the application of the SGPA RA algorithm in a massive CA setting, an example case where $K=30$ UEs are served via a single cell utilizing up to $M=$ $50 \mathrm{CCs}$, each with $N=100 \mathrm{RBs}$ is considered. Note that for $M=50$, the optimal RA problem formulation consists of $K M N=150,000$ binary valued variables $\left\{\alpha_{k, m, n}\right\}$. The normalized maximum transmission rate (link capacity) was considered as the utility function [5], [9], i.e.,

$$
\phi_{k, m, n}=\frac{1}{N} \log _{2}\left(1+g_{k, m, n} \mathrm{SNR}_{k, m}\right), \forall k, m, n,
$$

where the elements of $\left\{g_{k, m, n}\right\}$, representing channel gains, are independent, identically distributed (i.i.d.) according to an exponential p.d.f. of unit mean, and the elements of $\left\{\mathrm{SNR}_{k, m}\right\}$, representing the average CC signal-to-noise-ratios per UE, are i.i.d. according to a uniform p.d.f. over the interval $[-10,20]$ (in $\mathrm{dB})$. Note that for this choice of utility function and with $w_{k}=1$ for all $k$, the WSU is equal to the sum capacity of the system.

In all cases, the SGPA algorithm was initialized as $\alpha_{k, m, n}^{(0)}=$ $1 / K, \beta_{k, m}^{(0)}=1 / M_{k}, \gamma_{m}^{(0)}=1 / M_{0}$, for all $k, m, n$, and was restricted to perform 20 iterations irrespective of the RA problem dimension. Since convergence has not been achieved at this point in general, quantization of the final RA variables is performed, obtained by setting, for each $k \in \mathcal{K}$, the largest $M_{k}$ values of $\left\{\beta_{k, m}^{(20)}\right\}_{m \in \mathcal{M}}$ equal to 1 and zero, otherwise. The quantization of $\left\{\alpha_{k, m, n}^{(20)}\right\}$ and $\left\{\gamma_{m}^{(20)}\right\}$ is performed similarly.

For comparison purposes, a heuristic RA algorithm of comparable complexity with the SGPA RA algorithm was considered, consisting of two steps. First, by assuming $\alpha_{k, m, n}=1$ 


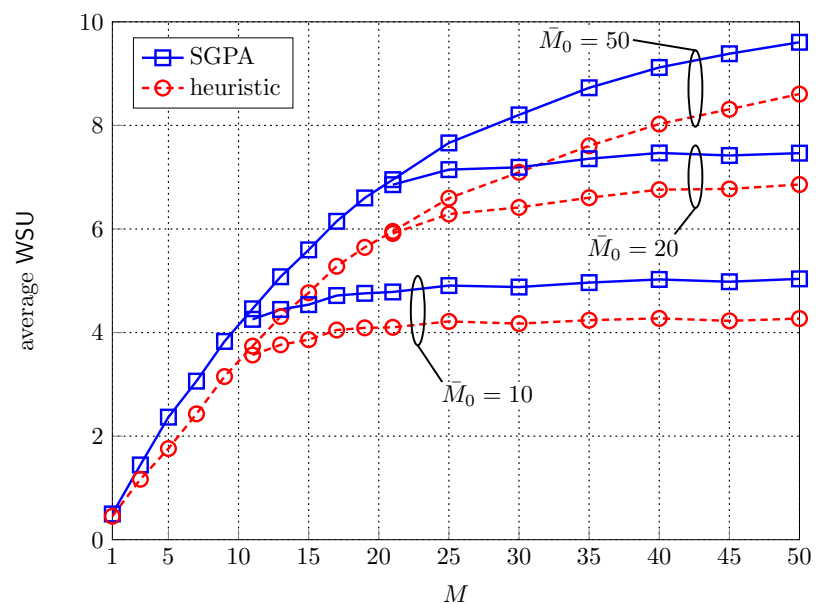

Fig. 2. Average WSU performance of RA algorithms with varying $M$.

for all $k, m, n$, it solves the relaxed RA problem w.r.t. $\left\{\beta_{k, m}\right\}$ and $\left\{\gamma_{m}\right\}$ after re-formulating it as a relaxed LP problem by introduction of auxiliary variables [12, Sec. 3.4]. After quantization of the LP solution with the same method as in the SGPA RA algorithm, the $\left\{\alpha_{k, m, n}\right\}$ variables are determined by the rule of (3) with $w_{k} \gamma_{m} \beta_{k, m} \phi_{k, m, n}$ in place of $w_{k} \phi_{k, m, n}$.

Fig. 2 shows the WSU obtained by averaging over independent realizations of utilities $\left\{\phi_{k, m, n}\right\}$ and UE weights, with the latter uniformly distributed over the set $\left\{w_{k}, k \in\right.$ $\left.\mathcal{K}: w_{k} \geq 0, \forall k, \sum_{k \in \mathcal{K}} w_{k}=1\right\}$. The maximum number of CCs per UE was set to $M_{k}=2$ for all $k \in \mathcal{K}$, whereas the maximum number of CCs used by the system was set to $M_{0}=\min \left\{M, \bar{M}_{0}\right\}$, with $\bar{M}_{0}=10,20,50$. It can be seen that, in all cases, increasing $M$ monotonically improves performance, since more resources are available for RA purposes. However, when $M_{0}$ is limited to a maximum value, consideration of $M>M_{0}$ provides only small gain, since the total number of available RBs for RA purposes remains the same. In comparison with the heuristic algorithm, the SGPA RA algorithm provides better performance by approximately $10 \%$ in all cases.

Fig. 3 depicts that average execution time of the SGPA and heuristic algorithms for the case shown in Fig. 2 corresponding to $\bar{M}_{0}=20$ (results are similar for other values of $\bar{M}_{0}$ ). A straightforward implementation in python was considered for the SGPA algorithm, while the LP solver of [13] was used for the heuristic algorithm. The measurements were performed on an Intel i5 core operating on Linux. It can be seen that both algorithms scale linearly with the problem dimension, which is essential for their practical implementation in massive CA applications. For the considered implementations, the SGPA algorithm is slower than the heuristic, which can be viewed as the price to pay for the improved performance. Note that the SGPA algorithm speed can be improved by reducing the number of performed iterations, with a cost in average WSU performance.

The effect of increasing the maximum CCs per UE is shown in Fig. 4, where the average WSU achieved via the SGPA algorithm is depicted for the case $M=50$. All UEs were

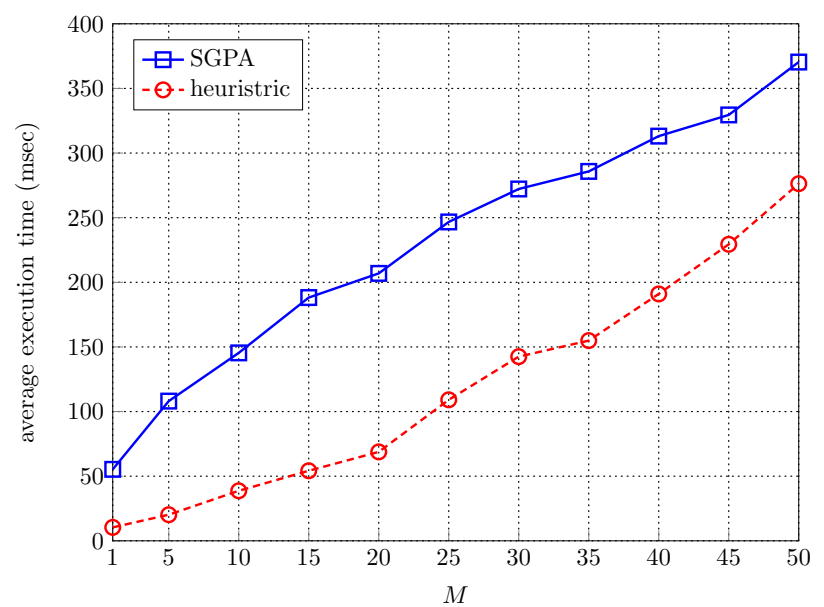

Fig. 3. Average execution time of RA algorithms with varying $M\left(\bar{M}_{0}=20\right)$.

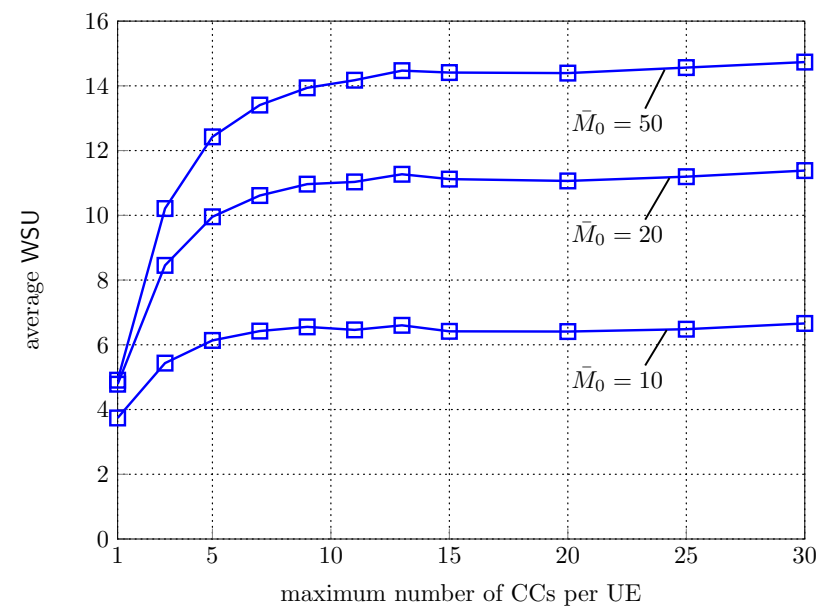

Fig. 4. Average WSU performance of the SGPA algorithm with varying maximum number of CCs per UE $(M=50)$.

set to have the same maximum number of CCs. As expected, increasing the maximum number of CCs per UE increases performance. However, this increase is substantial only up to a moderate number of CCs, above which, the ability of UEs to transmit to more CCs offers marginal gains. This is an interesting observation as it suggests that the cost associated with implementing devices able to communicate with multiple CCs is unnecessary for leveraging the benefits of massive CA.

\section{CONCLUSION}

An efficient iterative RA algorithm was proposed for massive CA scenarios. It was shown that the algorithm obtains the optimal RA solution under special cases of the RA problem, converges to binary-valued RA variables in the general case under mild assumptions on the distribution of utilities, and outperforms heuristic RA schemes.

\section{APPENDIX A \\ PROOF OF PROPOSITION 2}

For simplicity and without loss of generality, the case where $P_{0}=P$ will be considered, i.e., $r_{p}^{(i)}>0$ for all $p \in \mathcal{P}$. The 
following result is critical as it shows that at any stage of the iterations procedure before a fixed point is reached, one of the sequences $x_{p}^{(i)}$ is monotonically increasing towards the limit 1.

Lemma 4. Consider the RA algorithm initialization and the ordering of $\left\{r_{p}^{(i)}\right\}$ as described in the statement of Proposition 2 Assume that at iteration $i-1, i \geq 1$, the RA algorithm has not reached a fixed point and let $\mathcal{L}^{(i-1)} \triangleq\left\{p \in \mathcal{P}: x_{p}^{(i-1)}=\right.$ 1\}. Let $p^{*}$ denote the sequence index such that $x_{p^{*}}^{(i-1)}<1$ with $r_{p^{*}}^{(i-1)}>r_{p}^{(i-1)}$ for all $p \neq p^{*}, p \notin \mathcal{L}^{(i-1)}$. It holds

1) $x_{p}^{(j)}=1$ for all $j \geq i$ and $p \in \mathcal{L}^{(i-1)}$ with $r_{p}^{(i-1)}>$ $r_{p^{*}}^{(i-1)}$

2) Sequence $x_{p^{*}}^{(j)}, j \geq i$, is strictly monotonically increasing towards the limit value of 1 .

Proof: First note that it must hold $\left|\mathcal{L}^{(i-1)}\right|<L$, where $\left|\mathcal{L}^{(i-1)}\right|$ is the cardinality of $\mathcal{L}^{(i-1)}$, since, otherwise, the iterations would have reached the limit point where $x_{p}^{(j)}=$ $1, p \in \mathcal{L}^{(i-1)}$ and $x_{p}^{(j)}=0, p \notin \mathcal{L}^{(i-1)}$, for all $j \geq i-1$. In addition, note that the iteration scheme of (8) guarantees that, with $x_{p}^{(0)}>0, x_{p}^{(i)} \in(0,1]$ for all $i \geq 0$ such that $\left|\mathcal{L}^{(i)}\right|<L$. Therefore, index $p^{*}$ exists. Let $\mathcal{L}_{*}^{(i-1)} \triangleq\left\{p \in \mathcal{L}^{(i-1)}\right.$ : $\left.r_{p}^{(i-1)}>r_{p^{*}}^{(i-1)}\right\} \subseteq \mathcal{L}^{(i-1)}$. The normalization factor $\kappa^{(i)}$ of (8) is found as the unique solution of (97). It is easy to verify that $\left\{9\right.$ can only be satisfied if $\kappa^{(i)} \leq \min \left\{r_{p}^{(i)}\right\}_{p \in \mathcal{L}_{*}^{(i-1)} \text {. This }}$ condition implies from 8 that $x_{p}^{(i)}=1$, for all $p \in \mathcal{L}_{*}^{(i-1)}$. By repeating this argument for the next iterations, it follows that $x_{p}^{(j)}=1, j \geq i, p \in \mathcal{L}_{*}^{(i)}$, thus proving the fist claim.

Towards proving the second claim, note that $\kappa^{(i)}$ should satisfy either $\kappa^{(i)} \leq x_{p^{*}}^{(i-1)} r_{p^{*}}^{(i)}$ or $\kappa^{(i)}>x_{p^{*}}^{(i-1)} r_{p^{*}}^{(i)}$. In the former case, $x_{p^{*}}^{(i)}=1$, and by the previous argument, it follows that this value remains fixed for all subsequent iterations. In the latter case, $x_{p^{*}}^{(i)}=x_{p^{*}}^{(i-1)} r_{p^{*}}^{(i)} / \kappa^{(i)}$, whose value can be lower bounded by finding an upper bound for $\kappa^{(i)}$. Towards this end, it follows from (9) that

$$
\begin{aligned}
L & =\sum_{p \in \mathcal{L}^{(i-1)}} \min \left\{1, \frac{r_{p}^{(i)}}{\kappa^{(i)}}\right\}+\sum_{p \notin \mathcal{L}^{(i-1)}} \frac{x_{p}^{(i-1)} r_{p}^{(i)}}{\kappa^{(i)}} \\
& \leq\left|\mathcal{L}^{(i-1)}\right|+\frac{1}{\kappa^{(i)}} \sum_{p \notin \mathcal{L}^{(i-1)}} x_{p}^{(i-1)} r_{p}^{(i)} .
\end{aligned}
$$

Therefore,

$$
\begin{aligned}
\kappa^{(i)} & \leq \frac{\sum_{p \notin \mathcal{L}^{(i-1)}} x_{p}^{(i-1)} r_{p}^{(i)}}{L-\left|\mathcal{L}^{(i-1)}\right|} \\
& =\frac{r_{p^{*}}^{(i)}\left(x_{p^{*}}^{(i-1)}+\sum_{p \notin \mathcal{L}^{(i-1)} \backslash p^{*}} x_{p}^{(i-1)} \frac{r_{p}^{(i)}}{r_{p^{*}}^{(i)}}\right)}{L-\left|\mathcal{L}^{(i-1)}\right|} \\
& \stackrel{(a)}{<} \frac{r_{p^{*}}^{(i)}\left(x_{p^{*}}^{(i-1)}+\sum_{p \notin \mathcal{L}^{(i-1)} \backslash p^{*}} x_{p}^{(i-1)}\right)}{L-\left|\mathcal{L}^{(i-1)}\right|} \\
& \stackrel{(b)}{=} \frac{r_{p^{*}}^{(i)}\left(L-\sum_{p \in \mathcal{L}^{(i-1)}} x_{p}^{(i-1)}\right)}{L-\left|\mathcal{L}^{(i-1)}\right|}
\end{aligned}
$$

$$
\stackrel{(c)}{=} r_{p^{*}}^{(i)},
$$

where the assumption $r_{p^{*}}^{(i)}>r_{p}^{(i)}$ for all $p \neq p^{*}, p \notin \mathcal{L}^{(i-1)}$, was used in $(a),(b)$ follows from 9 evaluated at iteration $i-1$ and $(c)$ follows since $x_{p}^{(i-1)}=1$ for $p \in \mathcal{L}^{(i-1)}$. Therefore, $x_{p^{*}}^{(i)}>x_{p^{*}}^{(i-1)}$, i.e., $x_{p^{*}}^{(i)}$ is an increasing bounded sequence, which means that it has a limit $\bar{x}_{p^{*}}$. It follows from (8) that this limit must satisfy the condition

$$
\bar{x}_{p^{*}}=\min \left\{1, \bar{x}_{p^{*}} \lim _{i \rightarrow \infty}\left(r_{p^{*}}^{(i)} / \kappa^{(i)}\right)\right\} .
$$

Noting from 12 that $r_{p^{*}}^{(i)} / \kappa^{(i)}>1$, for all $i$, it follows that $\bar{x}_{p^{*}}=1$ is the only positive value that can satisfy $(13)$.

The proof of Proposition 2 now directly follows by noting that at iteration 1 there will exist an index $p^{*}$ such that $x_{p^{*}}^{(i)}, i \geq$ 1 , will be strictly monotonically increasing towards the limit 1 , according to Lemma 4 Therefore, for any $\epsilon>0$, there exists an iteration index, say, $i_{1}$, such that $x_{p^{*}}^{\left(i_{1}\right)} \geq 1-\epsilon$. When $\epsilon$ equals the finite precision used in the implementation of the algorithm, $x_{p^{*}}^{\left(i_{1}\right)}$ will be set equal to 1 . By Lemma $4, x_{p^{*}}^{(i)}=1$ for all $i \geq i_{1}$ and a new index $q^{*}$ will exist such that $x_{q^{*}}^{(i)}$ will start to monotonically increase towards 1 . It is easy to see that this procedure repeats until all sequences of index $p$ such that $r_{p}^{(i)} \geq r_{\pi(L)}^{(i)}$ will have achieved the value of 1.

\section{REFERENCES}

[1] K. I. Pedersen, F. Frederiksen, C. Rosa, H. Nguyen, L. Garcia, and Y. Wang, "Carrier aggregation for LTE-advanced: functionality and performance aspects," IEEE Commun. Mag., vol. 49, no. 6, pp. 89-95, Jun. 2011.

[2] A. Bhamri, K. Hooli, and T. Lunttila, "Massive carrier aggregation in LTE-Advanced Pro: impact on uplink control information and corresponding enhancements," IEEE Commun. Mag., vol. 54, no. 5, pp. 92-97, May 2016.

[3] 3GPP RP 142286, "LTE Carrier Aggregation Enhancement Beyond 5 Carriers", Mar. 2014; http://www.3gpp.org

[4] H. Lee, S. Vahid, and K. Moessner, "A survey of radio resource management for spectrum aggregation in LTE-advanced," IEEE Communications Surveys Tutorials, vol. 16, no. 2, pp. 745-760, 2014.

[5] F. Wu, Y. Mao, X. Huang, and S. Leng, "A joint resource allocation scheme for OFDMA-based wireless networks with carrier aggregation," IEEE Wireless Communications and Networking Conference (WCNC), pp. 1299-1304, Apr. 2012.

[6] Y. Wang, K. I. Pedersen, T. B. Sørensen, and P. E. Mogensen, "Carrier load balancing and packet scheduling for multi-carrier systems," IEEE Trans. Wireless Commun., vol, 9, no. 5, pp. 1780-1788, May 2010.

[7] Z. Huang, Y. Ji and B. Zhao, "An efficient resource allocation algorithm with carrier aggregation in LTE Advanced systems," in Int. Conf. on Wireless Communications \& Signal Processing (WCSP), Huangshan, 2012.

[8] M. Chiang, C. W. Tan, D. P. Palomar, D. O'Neill, and D. Julian, "Power control by geometric programming," IEEE Trans. Wireless Commun., vol. 6, no. 7, pp. 2640-2651, Jul. 2007.

[9] X. Wang and G. Giannakis, "Resource allocation for wireless multiuser OFDM networks," IEEE Trans. Inf. Theory, vol. 57, no. 7, pp. 4359-4372, Jul. 2011.

[10] S. Boyd and L. Vandenberghe, Convex Optimization. Cambridge University Press, 2004.

[11] R. Gunning and H. Rossi, Analytic Functions of Several Complex Variables. Prentice-Hall Inc., Englewood Cliffs, N.J., 1965.

[12] D.-S. Chen, R. Batson, and Y. Dang, Applied Integer Programming Modeling and Solution. John Wiley \& Sons, 2010.

[13] A. Domahidi, E. Chu, and S. Boyd, "ECOS: An SOCP solver for embedded systems," European Control Conference, pp. 3071-3076, 2013. 